\title{
A large intra-abdominal leiomyoma with unusual urinary symptom: case report
}

\author{
(1)Zafer Şenol \\ Sultan Abdulhamid Han Training and Research Hospital, Department of General Surgery, İstanbul, Turkey
}

Cite this article as: Şenol Z. A large intra-abdominal leiomyoma with unusual urinary symptom: case report. Anatolian Curr Med J 2022; 4(1); 120-122.

\begin{abstract}
Abdominal or intra-abdominal masses are defined as any enlargement or swelling localized in the abdominal area. Intraabdominal masses can be diagnosed from abdominal symptoms or through physical examination, as well as can incidentally be detected from radiological imaging performed for other reasons. In this study, the diagnosis and treatment of intraabdominal mass in a 27-year-old nulliparous female patient with unusual urinary symptom is presented. Preoperative magnetic resonance (MRI) images showed an intra-abdominal solid mass. The mass excision was performed, and surgical recovery was achieved. Pathological results revealed that the mass was a large leiomyoma of $15 \mathrm{~cm} \times 14 \mathrm{~cm} \times 11 \mathrm{~cm}$ size with extrauterine localization, which is quite rare in the literature. Post-operative results showed that the patient's existing urinary complaint had completely disappeared.
\end{abstract}

Keywords: Intra-abdominal mass, leiomyoma, urinary symptom

\section{INTRODUCTION}

Abdominal or intra-abdominal mass is any enlargement or swelling in the abdomen. Abdominal masses may be caused by hepatomegaly, splenomegaly, a retroperitoneal mass, a pancreatic mass, or omental metastasis (1). Abdominal pain, weight loss, notice of abdominal mass, fever, hematuria, dysuria, and intestinal complaints can be considered as various symptoms of the abdominal masses. In some cases, especially those deeply retroperitonal or pelvic localized may go unnoticed by the patient. Abdominal masses may have different pathological features in solid or cystic, benign, or malignant properties. It may also vary depending on age, gender, localization, and the source of tissue. Leiomyomas are the most common benign tumors in the female reproductive system, observed in $20-30 \%$ of women of reproductive age. Extrauterine leiomyomas, on the other hand, are quite rare, usually benign and can occur in any area of the body. Unusual growth patterns of such leiomyomas can even mimic malignancy and cause a clinical dilemma $(2,3)$. In this study, a large case of intra-abdominal mass, which was diagnosed as leiomyoma in pathology, and which unusually causes urinary symptom, is presented.

\section{CASE}

A 27-year-old nulliparous female patient was admitted to our hospital with a history of two years of frequent urination complaint. She was diagnosed with intraabdominal mass, which causes urinary symptom by pressing onto the urethra. Pre-operation Magnetic Resonance (MRI) images showed that the size of the solid mass was $17.7 \mathrm{~cm} \times 17.3 \mathrm{~cm} \times 7.8 \mathrm{~cm}$, and it was localized in the superior of the bladder and anterior of the uterus and showed an extension to the umbilicus superior (Figure 1). It has also been reported that the mass was in contact with the right ovary and histopathological evaluation is recommended.

Intra-abdominal mass excision was performed, surgical recovery was achieved, and the treatment was completed. Images of the mass excised from the patient are shown in Figure 2.

According to pathological results, a large leiomyoma with dimensions of $15 \mathrm{~cm} \times 14 \mathrm{~cm} \times 11 \mathrm{~cm}$ was diagnosed. In the post-operative follow-up, the preoperation urinary complaints of the patient disappeared completely. 

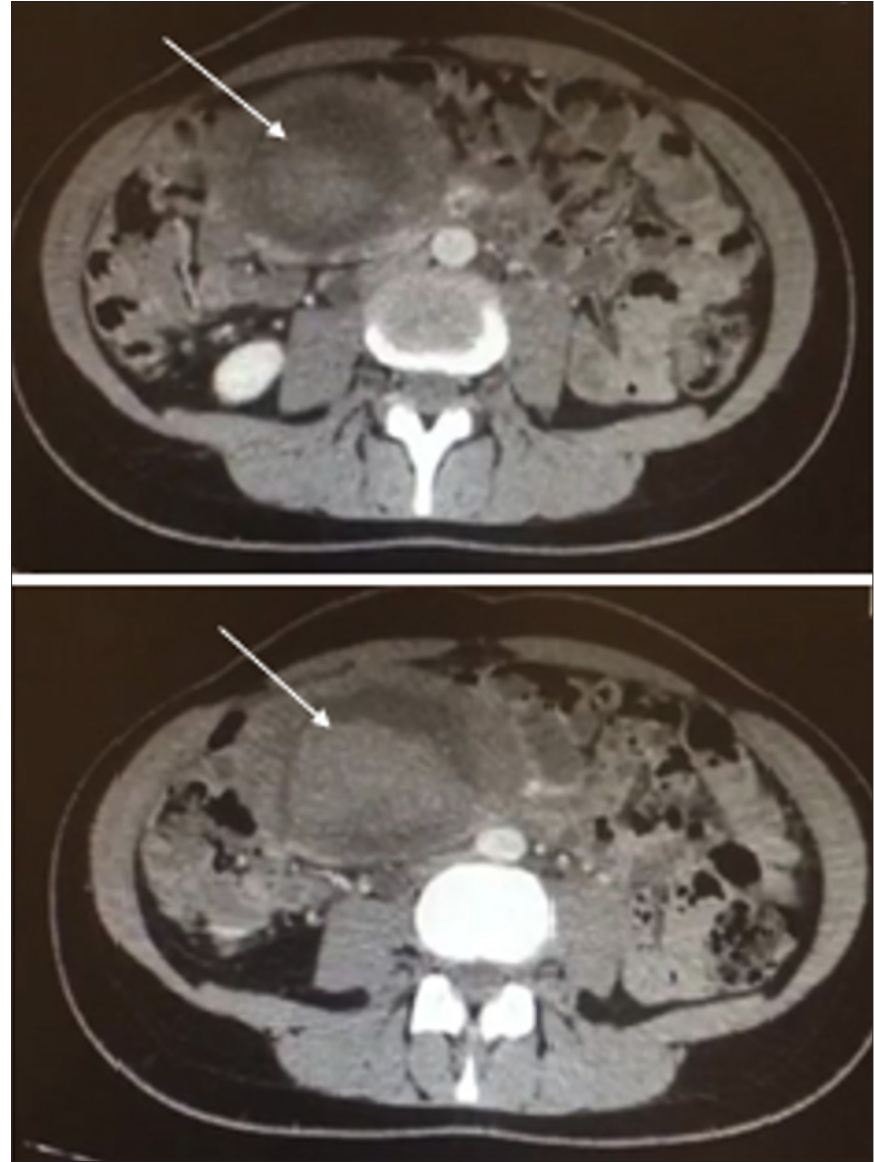

Figure 1. Magnetic Resonance (MR) images of the mass

\section{DISCUSSION}

Intra-abdominal masses can be evaluated with knowledge of abdominal anatomy. In the basic approach, there are nine regions of the abdomen: umbilical, epigastric, suprapubic, right and left hypochondrium, right and left lumbar, right and left inguinal regions. In the clinical practice of surgeons, the regions of the abdomen are evaluated as right and left upper and lower quadrants, epigastric and hypogastric regions (4). Detection of intra-abdominal masses can be performed by physical examination findings due to symptoms or incidentally during radiological imaging for different reasons. For investigation of abdominal masses, several radiological imaging can be used such as X-ray radiography, abdominal ultrasonography (USG), magnetic resonance imaging (MRI), and computed tomography (CT) method (5). MRI is useful for imaging of the abdomen in cases with pancreatic, retroperitoneal masses, patients with renal insufficiency, or in patients for whom the use of contrast agents containing iodine is a contraindication (6). The diagnosis of an intra-abdominal mass consists of two stages which are a detailed anamnesis: an assessment of the patient's history and a physical examination. Evaluation of the patient's history includes the symptoms and the time since the onset of symptoms, past illnesses, family history, and, if any, the patient's previous surgeries

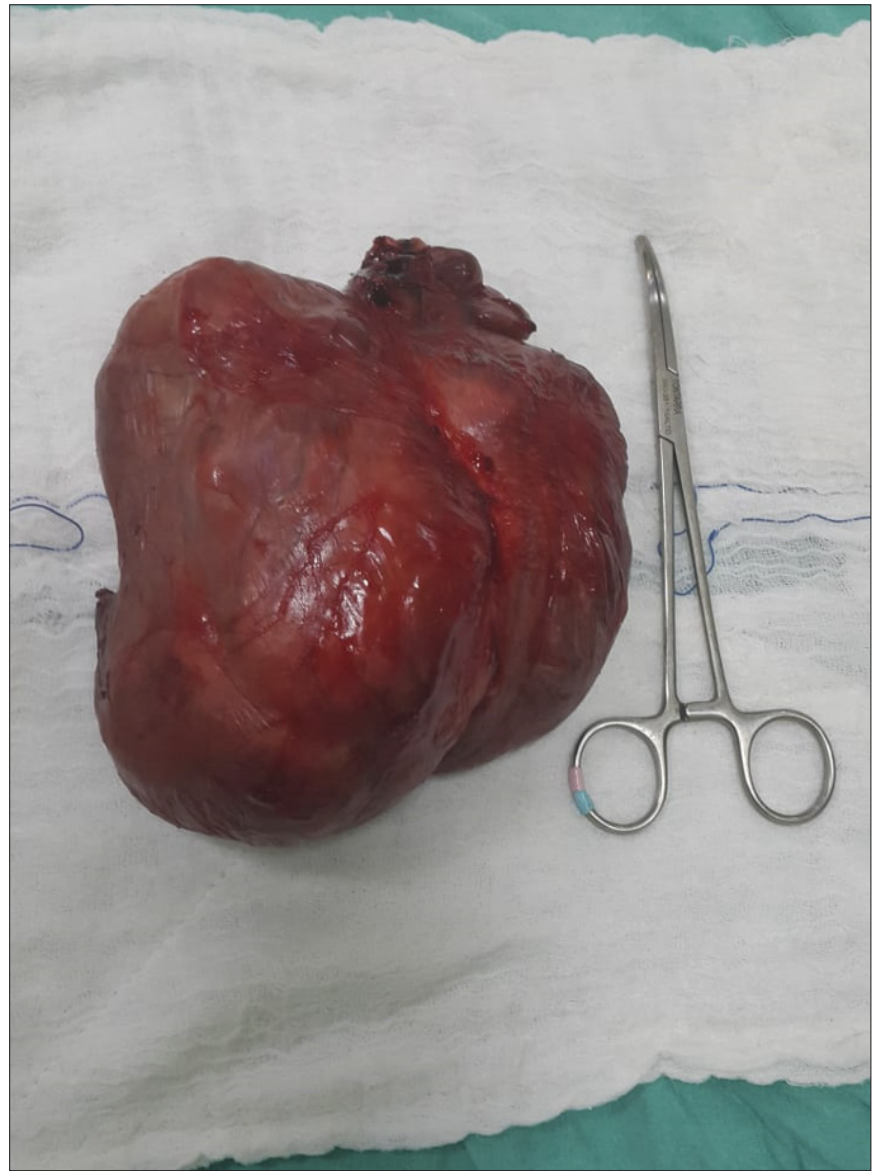

Figure 2. Image of intra-abdominal leiomyoma $(15 \mathrm{~cm} \times 14 \mathrm{~cm} \times 11$ $\mathrm{cm}$ ) excised from the patient

(7). Some abdominal masses that are not very large in size may remain asymptomatic or cause abdominal pain in some cases. In some cases, it may be difficult for the patient to notice the masses, especially in those that are located deep retroperitoneal or pelvic (8). Leiomyomas are benign tumors that occur in the female reproductive system. In leiomyomas, surgery is the main treatment approach and is curative. Extrauterine leiomyomas have been reported quite rarely in the literature (9).

\section{CONCLUSION}

The case presented in this study is a large leiomyoma with an extrauterine location detected in the intra-abdominal region and causing unusual urinary symptom, which is quite rare in the literature. The postoperative results of the patient showed that all urinary symptoms had disappeared, and surgery was curative in the treatment. Consequently, urinary complaint should be considered in the differential diagnosis of intra-abdominal leiomyomas.

\section{ETHICAL DECLARATIONS}

Informed Consent: Written informed consent was obtained from all participants who participated in this study. 
Referee Evaluation Process: Externally peer-reviewed.

Conflict of Interest Statement: The authors have no conflicts of interest to declare.

Financial Disclosure: The authors declared that this study has received no financial support.

Author Contributions: All of the authors declare that they have all participated in the design, execution, and analysis of the paper, and that they have approved the final version.

\section{REFERENCES}

1. Das S. Examination of abdominal lump: A manual on clinical surgery. $11^{\text {th }}$ ed. Calcutta: S.D. Publications; 2015.

2. Fasih N, Prasad Shanbhogue AK, Macdonald DB, et al. Leiomyomas beyond the uterus: unusual locations, rare manifestations. Radiographics 2008; 28: 1931-48.

3. Midya, M, Dewanda, NK. Primary anterior abdominal wall leiomyoma-a diagnostic enigma. J Clin Diag Res 2014; 8: NJ01-2.

4. Swartz, MH. Textbook of physical diagnosis: History and examination. 5th ed. Philadelphia: Saunders Elsevier; 2006.

5. Bhagat S, Gauba N, Singh S, Singh A, Mahal GBS. Assessment and comparison of abdominal masses by sonography and computed tomography. J Evol Med Dent Sci 2014; 3: 84-94.

6. Gascin CM, Helms CA. Lipomas, lipoma variants, and well differentiated liposarcomas (atypical lipomas): results of MRI evaluations of 126 consecutive fatty masses. Am J Roentgenol 2004; 182: 733-9.

7. Brady MS, Gaynor JJ, Brennan MF. Radiation associated sarcoma of bone and soft tissue. Arch Surg 1992; 127: 1379-85.

8. Akkoca, M, Tokgöz, S, Yılmaz, KB, Akıncı, M, Yılmazer, D. Diagnosis and treatment approaches for intraabdominal masses in adults. Ankara Üniv Tip Fak Mec 2017; 70: 201-5.

9. Nasta A, Nandy K, Bansod Y. An unusual case of abdominal leiomyoma presenting as a free lying intraperitoneal mass in an elderly gentleman. Case Rep in Surg 2016; 1714958. 